more likely to adhesions and cicatrices in the fauces.

A singer should be operated upon by a laryngologist who has some knowledge of the art of singing and who can operate with great skill and carry out careful postoperative treatment.

\section{ELLEN J. PATTERSON.}

Lewis, P. M.: Tonsillectomy Under Novocaine. Med. Rec., 1916, xc, 1116.

The author adrocates the use of novocaine in tonsil work only in adults of a phlegmatic temperament where the patient is given his choice of a local or general anesthetic.

The advantages of tonsillectomy under novocaine are that the patient sits in the upright position with the mouth open without mouth-gag or tongue-depressor, and can expectorate all blood; the operation is painless if the tissues are prop- erly anesthetized; hemorrhage is insignificant both during and after operation and the patient can go home alone immediately after the operation.

The technic is as follows: after anesthetizing the peritonsillar tissues with a 4 per cent. solution of cocaine, an application of a 10 per cent. solution is made to allay the pharyngeal and faucial re. flexes. A 2 per cent. solution of novocaine with a few drops of adrenalin is then injected into the tonsil, the anterior and posterior pillars, and the edges of the tonsil. Aifter the tonsillectomy onefourth to one-eighth grain of morphine is administered hypodermatically to check the hyperactivity of the salivary glands and decrease the tendency to frequent attempts at delutition with the idea of preventing hemorrhage.

ELLEN J. PATTERSON.

\title{
HEMATOGENOUS INFECTIONS DUE TO SEPTIC DENTAL FOCI.
}

\author{
By Dr. Mallory Catlett, Denver, Colo.
}

I am, indeed, appreciative of the honor of appearing before you at this time, and taking part in the discussion of the greatest subject in the history of dentistry. However, in the allotted time, I can discuss only in a superficial manner, a few phases of the subject; yet if I can drive home one thought which would lead to higher ideals and better dentistry, I will feel that my efforts have not been in vain.

The area of anatomy allotted to the dental profession to guard against disease, is very small, compared to that of the physician, but our possibilities, rival, if not exceed his in the prevention of disease, for preventive medicine, if effective, must include preventive dentistry, but with all the advancement that medicine, surgery and dentistry have made, the diseased conditions in the mouths of civilized people are said, by investigators, to be ten times worse than they were a hundred years ago; and with the multiplication of oral diseases, the systemic diseases resulting therefrom have increased proportionately, until today medical patients are seeking relief thru dental operations.

The results obtained by treating the oral lesions, has been so convincing as to origin, that today we are expected to restore to health, the gastro-intestinal sufferer, the neurtesthenic,-all forms of rheumatism, including septic arthritis, to benefit endarteritis, septic endocarditis, neuritis, anemia,-pernicious and otherwise, and functional disturbances of the eye, ear and brain; to cure and prevent Ludwig's Angina, and ferunculosis; to 
prevent tuberculosis, asthma, cancer and appendicitis, diseases of the kidneys, and innumerable other infections which a clean mouth would prevent. Thru the collaboration of eminent diagnosticians, with bacteriologists, many systentic diseases heretofore of unknown origin, are now known to be due to infection absorbed at some septic focus.

Infections occurring in any part of the body, other than at the point of entrance, are spoken of as Hematogenous infections (via blood stream.) Hematogenous infections, due to septic dental foci, would then be defined as any systemic disease, due to the absorption of pathogenic micro-organisms incubated at some septic dental focus, the duration of the systemic disease depending upon the permanency of the focus, and the virulency of the infecting organisms. Just as invading armies must have bases of supplies, lines of communication into, and become entrenched in the country of their enemies, so must micro-organisms have bases of supplies and avenues of entrance into and become entrenched in some vulnerable part of our anatomy, before any serious harm can result. Their base of supplies would be the septic dental focus, and avenues of entrance into the body is via circulatory fluids and gastro-intestinal tract.

Lest you forget-a septic dental focus may be defined as any acute or chronically inflamed area from the mildest form of marginal gingivitis, thru all its stages, to a hopeless case of pyorrhea; any acute or chronic septic apical pericementitis; alveolar necrosis; suppurations around partially or completely impacted teeth; any gingival inflammation, due to mechanical, chemical, or bacterial irritants-as fillings, crowns, bands, bridges, partial plates, food impactions in interdental spaces, malocclusions, bac. terial placques, fermenting food debris, strong medicine; and escharotics applied either to apical tissues or to gingivae. In mouths with any of the foregoing patho- logical lesions, micro-organisms find ample pabulum.

From these sources the bodies of our patients become slowly but surely impregnated with pathogenic germs and their toxins, and the premature deaths resulting therefrom, I believe, to be greater than epidemics, famine or war. I am not especially interested in what individual organism, or combination of organisms, are responsible for these diseases, nor thru what morphylogical transmutations they undergo. Inquisitive internists have brought to light the fact that pus and foreign bodies wherever located, are alarming symptoms so far as the health of the patient is concerned. of all locations for pus to be present, I believe we can least afford to have it in our mouths. What would you expect the results to be if you have a patient with six or eight chronic abscesses around their finger nails and all pus from these abscesses were allowed to be swallowed and furnished to muscle, nerve and bone cells as food? This is what is allowed in hundreds of thousands of patients frequenting dental offices annually.

This fact should be recognized by dentists. I have been closely associated with medical men ever since graduation, having officed in a surgical hospital four years of the time, and I am thoroly convinced that mutual cooperation and consultation is essential, beneficial equally to one as the other. Patients are being dismissed daily with the assurance that all is well, with abscess after abscess and peridental inflammations galore. The supposition, long held, that all ingested germs were destroyed by the gastric juice, is no longer entertained.

Rosenow has shown that bacteria seemingly benign while exposed to oxygen, may become extremely virulent after being taken into distant parts of our bodies, as muscles, tendons, joints, valves of heart, lungs, kidneys, etc., and their supply of oxygen lessened. It may take years and years of absorption before any systemic disease is evidenced, depending en- 
tirely upon number of foci, the virulence of infecting organisms, and the individual resistance; but give them time, and they are sure to cause trouble, taking advantage to assert themselves during periods of depressed vitality.

These theories are not at all new to the dental profession. D. D. Smith, Miller, Black, Talbot, Hartzell and others have long held that many grave, systemic diseases were due to oral sepsis. Adami speaks of the possibility of infection from a chronically inflamed area, as that around crowns, raw surfaces beneath bridge dummies, chronic gingivitis, etc.; stating that the phagocyte passes entireIy to the outer surface of these areas, and then re-enters the circulation, laden with pathogenic germs. The possibility of apical infections via this route is highly probable.

Gentlemen, we are confronted with the cold facts, that peridental inflammation, beginning both at apex and gum margin, are causing serious systemic diseases; also the possibility of systemic infections, following absorption from any chronically inflamed surface. These facts we cannot deny. What is done when a serious contagion threatens any part of our country? It is stamped out at once and vigorous prophylactic measures inaugurated against its recurrence. If disease or foe threaten from a foreign country rigid quarantine and defenses are established at the ports of entrance-not in these Rocky Mountains, but at the water front.

The question of national preparedness pales in insignificance, compared to the benefits derived by defending our bodies against invasion by hosts of pathogenic organisms. So we should begin at the portal of entrance, and render the oral cavity as immune to disease breeding germs, as possible, by a combination of scientific, sanitary, mechanical, aseptic, surgical and prophylactic procedures. There is usually due and timely notice of approaching disease to prevent a great proportion, but our diagnostic ability is so lacking, that very frequently benign lesions develop into serious conditions, before it is deemed even necessary to apply any remedy. It is just as sane to. wait until pulpitis or putrescence occurs, to fill a cavity, as it is to wait until pyorrhea is fully established, to apply any treatment.

In order to thoroly appreciate and understand these pathological lesions one should have an intelligent foundation upon anatomy, histology, physiology and general pathology, combined with embryclogy and bacteriology. If we were to devote the time spent idly, in a careful study of these subjects, our knowledge would be so sound, that when a physician referred a patient, suffering with systemic complications, and possibility of dental origin, our diagnostic information would be so accurate we would be able to give him a detailed, scientific report of our pathological findings, checked up with clear radiographic proofs; and furthermore, our advice to the patient, would be so convincing, few would decline to accept it. Remember, you are the control between physician and patient, and your diagnosis should not be haphazarda careful study should be made of all suspicious areas, with particular scrutiny of all inflamed surfaces, for it has been my experience that systemic diseases, resulting from peridental inflammations far outnumber those resulting from periapical involvement, for in many cases there are no pulpless teeth.

The apical abscess is circumscribed and walled off, while peridental infections are not limited. Remember tho, that all apical abscesses are blind abscesses, except when in active discharge; that it is only at times when more pus is generated than nature can absorb, that it ever evacuates by way of fistulus opening. I cannot imagine a more menacing source of infection than a well established case of pyorrhea. Remember that gingivitis, either continues as a chronic inflammation, or ter minates in suppuration and, loss of 
teeth; never in resolution. The radiograph is indispensible, and should be studied carefully, and compared with clinical symptoms, giving the health of the patient first consideration over value of teeth.

All septic food must be eliminated, and after you are convinced that all are removed, if the patient's general condition does not improve, you have assisted in diagnosis by exclusion, and rendered invaluable service to the patient. No better diagnostic symptom could occur, however, than to have the systemic condition become immediately worse after local treatment, due to freshly opened channels for infection. To avoid this, make 20 minute appointments, twice a week.

Do not take it for granted that you can locate the cause of trouble, by reason of the fact that an M. D. referred the case. The teeth may be o. k., tho he may tell you that every other source has been exhausted, and it may have been, according to his ability. Many are too quick to suspect the teeth, while others never give teeth a thought and continue treating symptoms. I have frequently seen patients, who have been sick for months and years, with enough pus and filth in their mouths to kill them, and their physicians and consultants had never looked into their mouths. So establish your own case, and do not hesitate to give either an affirmative or negative opinion as to cause.

Frequently patients come to me with this history- I have had a dentist for a great many years, he has done lots of fine work for me, but my teeth are loose and sore. I am sick, have no appetite, am as tired in the morning as at night, have no energy. My dentist says he thinks I have pyorrhea, but nothing can be done for it; to tolerate it as long as I can, and then have them out. I can make excuses for the man who makes a mistaken diagnosis, and removes a pulp from a vital tooth, in treating a pericemental abscess; or one who continues to scale a pyorrheal tooth; with a flow of pus coming from a putrescent pulp, via apical foramen; but I cannot excuse anyone who gives such advice, or one who does not warn his patience of the approach of a slowly progressing destructive disease, which will surely result in loss of teeth, and often even the health of patient.

There are several things that can be done. First and best of all, familiarize yourself with the knowledge and with digital skill, necessary to prevent such conditions; second, extract the teeth when they seem to get beyond you; or third, send them to someone who will do something for them, for when conditions reach this stage, they will quit you and quit you for good, So do one of these things, before the patient's health is impaired. One thing, I believe, responsible to a great extent for these conditions, is, that we have too many patients to do justice to any, and select the kind of work we like best, and let the rest go, and soon all is lost. Another thing responsible, too, is the careless way work is done. When I heard Dr. A. D. Black say that the majority of all inflammation in the mouths of young adults, between ages of 20 and 35 , who frequent dental offices, was due to defective dental work, I thought him mistaken, now I fully agree.

The more crown and bridge work I see the more convincing is Hunter's condemnation. Schemgurg states that most all of the work done by oral surgeons is made necessary by defective dental operations. These are true, stinging indictments. I believe you will agree with me that at least $70 \%$ of adult teeth lost, are lost from peridental diseases, beginging at the gum margin. Then, why so much more consideration given the prevention of the other $30 \%$ loss? We have about arrived at the time when there should be less talk of pyorrhea pockets, loose and pulpless abscessed teeth; but more of prevention, such as outlined and successfully practiced by D. D. Smith, 
Pickerill, Russell W. Bunting, and many other specialists and clinicians thruout the country.

The report of the following cases should be convincing as to the origin:

Case 1.-Mr. D., 35. Had not been free from boils for four years. Been on rigid diet, but no appetite, and no energy. Had taken medical treatment in Washington, $D$. C., including autogenous vaccines. Referred to dentist-gave negative report, altho he gave him the emetin treatment to prevent pyorrhea. Moved to Colorado and physician referred him to me in January this year. Pus found around 17 teeth. By persistent treatment this was eliminated, and since March 4th has been entirely free from boils; eats and feels well.

Case 2.-Miss C. M. G., 32, volunteered visit to my office, Sept. 6, 1912, before going to hospital for operation for gastric ulcer. History of stomach trouble for three years. Visited different health resorts, and consulted many diagnosticians, all hopes of relief abandoned, except surgical operation. After the extraction of one pyorrhetic molar, and treating rest of mouth, all symptoms of gastric ulcers gradually subsided. No operation was performed. Diet of soda crackers and milk was continued for three weeks, when other things were added. In three months she was eating everything and had gained 14 pounds, normal weight 145 pounds, weight when pyorrhea work begun, 111 pounds. Report in August, 1915, no return of trouble. Weight, 156 at present.

Case 3.-42, Miss B., bank employe, Sioux City, Ia. Septic Arthritis, both knees. Trouble began as screpitis which lasted one month, when pain and swelling appeared. All known rheumatic remedies given, without beneficial results. Her Iowa dentist gave her a clean bill of dental health. Advised to come to Colorado, and on Aug. 15th, Dr. C. R. Lindsey was called to hospital to make dental examination - she not being able to walk, except with aid of canes. Found inflammation around four malfitting crowns. Crowns removed, one molar with perforation extracted, teeth scaled and polished, and referred to me for completion. Canals of five pulpless teeth were filled, M. O. D. inlays placing instead of recrowning and bridge put in with M. O. D. inlays on vital abutments. By Dec. 27th she was well enough to walk without aid of canes, and February, 1916, letter stated she was $O$. K. and back at work.

Case 4.-A tuberculus, cadaveric looking gentleman, about 45 , height 6 feet 2 inches, weight 132, suffering intensely with inflammatory rheumatism, in tendons of feet and hands, and all over back. This gentleman had received treatment from most all medical specialists in Denver, including eye, ear, nose and throat, lung, G.-U. and general practitioners as well. Had autogenous vaccines from sputum and prostate gland; no benefit. Changed physicians and was referred to me latter part of July, 1915, and not a bad case of pyorrhea was noted; however, soon after beginning treatment, a large abscess developed on his back, which delayed treatment for three weeks, after which time, it was completed with a speedy recovery. By January, 1916, he was entirely well and had gained twenty pounds.

Class 5.-Lady 35. Severe neuralgic pains, referred to eye, ear, occipital region, neck and even chest, with extreme nervousness, and mental depression. Took treatment from M. D. for two months, and he referred her to nose and throat man, who in turn told her to go to her dentist, and she informed him that her dentist had told her that her teeth were not responsible, whereupon she visited another of her acquaintance, and he said, teeth not sufficiently bad to cause so grave a disturbance. I saw her March, 1915, and had to extract every upper molar-they being hopeless from pyorrhea, cervical caries and pulpstones. Almost immediately the neuralgia disappeared and in due time all was well. 
Case 6.-Is report of three cases of inflammatory rheumatism in women of about same age, 65 years. All had hopeless cases of pyorrhea, complicated with some apical infection. One case requires special mention, as it was seen in March, 1913, and advised to have the remainder of her teeth extracted (the lower anterior six, all that remained). However, they were retained for two years longer, when she was delirious for three weeks in February and March of 1915, with high fever and rheumatism around her heart. In April they were extracted, and from that date, she has had steady improvement and went thru last winter and spring with comfort. Both other cases recovered after extraction of pyorrhea teeth.

If time permitted, I could report many more interesting cases of recovery where medical treatment had entirely failed. In conclusion, I would advise that pulps be removed, except when exposed or infected, as at best, it is a hazardous undertaking, even in normal conditions; a perfectly filled canal is very rare. Guard against and remove all causes of gingivitis, realizing that by avoiding pulp removal, and the prevention of pus formation, you are removing the primary causes of systemic diseases and lengthening the lives of your patients.

Great deeds are being done by men of ambition and capacity for work in our profession. Great sacrifices are being made by the trustees of our Research Institute, in order that all dentists may better serve humanity. The greatest opportunity in history is dawning upon us. Our possibilities are limitless and our ambitions for achievements should fill us with virile determinations to do our level best. -Bulletin Colorado State Dental Association.

\title{
A DEVELOPING PROFESSION-HOW SHALL WE MEET THE OPPORTUNITIES AND RESPONSIBILITIES OF THE NEAR FUTURE? * (Abstract.)
}

\author{
By Edmund Noyes, D. D. S., Chicago, llinois.
}

The methods of making pulp canal operations as they should be made have been well and minutely described in a number of excellent papers. These have naturally enough dwelt most elaborately upon the difficult cases, and have had so much to say about working an hour or more over some fine canal and then trying some more at another sitting and have insisted upon from two to four or five X-rays in the course of the proceedings, at the same time requiring such elaborate arrangements for asepsis that I have feared a great many men, practicing for patients in very moderate circumstances, will throw up their hands in de. spair and say "that practice is not for

*April, 1917, Dental Review. us" and so be tempted to shirk their duty and responsibility in the matter and continue on in their same old way.

In the first place, then, nine-tenths of all the teeth except the molars and lower incisors can, if in normal condition, have their pulp canals cleaned out and filled without any serious difficulty and without consuming an unreasonable amount of time and the filling can be carried to the end of the pulp canal, and we can know that it is there and can fill the remainder of the canal without leaving any vacant spaces. It is to be remembered that the pulp canal terminates in the dentin and the passage thru the cementum at the end of the root is only a foramen and is usually very small. A 